\title{
Research on the development of Sports Anthropology
}

\author{
Wang Jun ${ }^{1 a}$, Su Yingying ${ }^{1 b *}$ \\ ${ }^{1}$ College of physical education, Yunnan Agricultural University, Kunming, China \\ Aemail : 158291721@qq.com, bemail : 963990848@qq.com, \\ * Corresponding Author
}

\section{Key words: Sports; Sports Anthropology; Discipline Orientation; Discipline Development}

\begin{abstract}
Anthropology is becoming more important than ever before in the context of Globalization. Moreover, there is a growing need to understand the relationship between individuals and society, also the similarities and differences with each other. This paper analyzes the characteristics and development trend of the discipline of Sports Anthropology, explore some problems in the development of discipline, and then induce the discipline orientation of sports anthropology based on trends in international and Chinese own actual situation.
\end{abstract}

\section{体育人类学学科发展研究}

王钧 $1 \mathrm{a}$, 孙芗瑛 $1 \mathrm{~b}$ *

1 云南农业大学体育学院, 昆明, 中国

Aemail: 158291721@qq.com : bemail: 963990848@qq.com,

*通讯作者

关键词：体育；体育人类学；学科定位；学科发展

摘要: 在全球化背景下, 人类学正变得前所未有地重要, 人们越来越需要了解彼此之间的相 同与不同，了解个人与文化之间的关系, 籍由阐释 “他者” 而达到对自我的认识。本文分析 了体育人类学学科发展及其特点, 探讨了学科发展的一些问题, 并结合国际上学科发展的情 况与我国的实际, 对体育人类学的学科定位进行了反思。

\section{1.体育人类学的学科发展及其特点}

体育人类学早在上世纪的欧美国家就已经得到关注, 经过几十年的发展已经确立了独立 的学科地位。它最初被定义为“原始运动”或“体育文化活动”的研究。随着现代人类学学科体 系的形成, 学科的研究领域趋于宽广, 体育人类学研究对象也更加多元, 延伸到现代社会的 各个方面。奥林匹克运动的崛起让国外的研究者把他们的研究视野扩展到现代体育, 主要关 注现代社会体育运动中的问题, 特别是探析体育运动中表现出的社会现象分析。随着全球化 的趋势许多社会的矛盾通过体育活动反映出来。这种大背景下体育人类学被重新定义为研究 人类的整体体育文化多样性, 研究的领域也从农村社会发展到现代城市, 从单一的活动发展 到地域整体文化。

体育人类学继承了传统的人类学研究方法, 但仍然专注于实地的考察, 尤其是参与性观 察。通过长期深入的田野调查, 不断挖掘区域体育文化的脉络。随着传统时代和社会环境的 快速变化, 体育人类学的研究主题也处于剧烈的变化中, 并出现了许多新的情况和问题 ${ }^{[1]}$ 。 
在这方面, 体育人类学的研究者们给予了充分的重视, 力图把握这些变化的趋势和规律, 了 解它对人们社会和文化生活的影响。与此同时, 随着社会, 经济和科技的不断发展, 一些新 的体育产品、体育现象油然而生, 如电子竞技, 文化安全, 广场舞蹈, 体育电影, 体育科技 等等。面对这些新的社会现象与问题, 体育人类学研究者迅速作出反应, 运用人类学的知识 和视角进行充分解读，彰显了体育学者对于文化变迁的敏感性和洞察力。

随着新领域的不断介入、新人类学分支学科的相继出现, 人类学理论的发展呈现处百家 争鸣的新常态。体育人类学研究者们也一改过去对宏观、普适理论的追求, 进而改为对真实 世界、真实问题、真实生活的探微研究。虽然体育人类学学科理论建立还相当稚嫩, 但是共 同的研究主题和研究方向仍是研究者们建立学术联系、寻求学术认同的途径。此外, 跨领 域、跨学科合作也逐渐打破原有单独学科生态成为体育人类学科的一种新发展常态。研究边 界的宽广，促使体育人类学在研究方法、研究范式和研究方向上产生了更多的可能性。

近 30 年来, 体育人类学在学科创建与发展中, 逐步形成了独特的领域特色与学科特 点:

（1）综合性研究的学科。体育人类学是综合性学科, 它依赖于社会科学、自然科学的研 究成果。它从人的生物属性和社会 (文化) 属性两大方面来研究体育的萌芽、成熟和发展演 变。体育人类学起源于近代自然科学和人文社会科学的交叉发展, 与文学、心理学、历史 学、哲学、生物学都有着密切的联系。从各国研究的情况看, 尽管各国依据不同的学科传统 而各有侧重, 但体质人类学、体育与民族文化认同、体育与宗教仪式、体育与性别、体育与 阶层、体育文化变迁仍是体育人类学的主要关注对象。体育人类学的研究范式更加关注对象 的整体性, 不但要将体育活动的本质属性与行为现象进行有机联系（包括参与者、社会、文 化，甚至心理)，更是从宏观视角审视特定文化生态环境与社会环境，从而共同整合一个围 绕体育的综合“生态系统”来加以研究。

（2）跨学科研究的学科。自体育人类学诞生以来，其学科设置就具备人文的上位属性与 杂糅的多学科色彩。体育是一个基于人类身体基础的文化现象, 体育学原本就是一个多元学 科相结合的综合学科, 生物学、医学、历史学、哲学、社会学、人类学等与体育学发生融合 交叉，而且相互渗透的程度日益加深。人类学家埃里克 R.沃尔夫（Eric R. Wolf）认为，人 类学是人文学科中最为科学的, 且是科学中最为人文的。[2]体育人类学亦是如此, 它的研究 领域通常具有人文学科的属性，但又常常沿袭人体科学的范式习惯。这种“桥式”学科的定 位, 是综合运用体质人类学和文化人类学的极佳结合点, 它既是人文学科的重要组成部分, 又具有极高的科学应用价值。随着研究的不断分化扩展, 体育人类学介于自然科学和人文学 科间的跨学科特性不断彰显, 从体育的视角出发, 既可以从生物和生态环境的角度对人类进 行研究, 也可以从社会和文化角度研究人类。

（3）反思性研究的学科。马林诺夫斯基曾指出：“抱有敬意地去真实了解他人（即使是野 蛮人）的基本观点.....无疑会拓宽我们认识的深度。如果我们不能搁置那些自生来便接受的 风俗、信仰和偏见的束缚, 我们便不可能达到苏格拉底那种 “认识自己” 的真正智慧” [3]。人类 学的传统是对异文化的研究，但并不止于此，人类学更加深层的学科旨趣在于“反求诸已”, 以他者之镜映照和反思对自身的认知，进而通达“人之所以为人”的本质。文化相对论的视角 和跨文化比较的方法共同构成了反思性研究的两大基本工具。人类学研究的一个基本目的就 是致力于端正我们人类的文化观，摆脱民族中心主义的观念或是消解文化比较中存在自卑 感, 并促进各民族文化进行身份平等的交流。体育人类学也是这种通过研究他者来反思自 我, 从体育文化生态的视角建立这种特殊的“自省”。再吸收与借鉴非西方社会人类学的研究 经验, 将我国的体育人类学建立起符合东方民族体育文化的认知体系。这种体系是从经济生 活、政治组织、亲属制度和宗教信仰四个方面对民族传统体育文化的整体性加以认识。虽然 体育人类学的研究对象日趋多元, 但是已经形成的文化认知体系与范式依旧是理解与阐释人 类学现象的基准框架。正是基于对“弱势”、“边缘”群体身体文化的深入阐释，体育人类学者 
通观人类体育的生存与发展趋势, 认识到亚洲各国丰富多彩的民间传统体育项目作为民族文 化宝藏, 对于弘扬东方体育文化价值, 促进世界体育文化多元, 发展人类所共有的体育事业 具有重要意义 ${ }^{[4]}$ 。显然, 这是一种超越了“传统与现代”、“落后与野蛮”二元对立巢臼的“各美 其美，美人之美”的文化自觉和文化自信。

\section{2. 中国体育人类学学科发展的问题}

一般而言, 学科发展水平主要体现为以下几个方面: 1) 学科理论体系是否成熟; 2) 学 科人才培养模式是否完善； 3 ）是否具有健全的学术组织；4）是否有代表性的系列学术著 作。目前我国体育人类学的发展面临着很多问题, 从学科发展现状来看, 主要存在着以下三 个主要问题:

\section{1 学科队伍薄弱与基础理论贵乏}

由于起步较晚以及学科定位的争议, 中国体育人类学学科发展至今仍然处于亟待完善的 阶段。全国仅华南师范大学、广州体育学院、厦门大学、华中师范大学、中央民族大学、宁 波大学等几所学校开设体育人类学相关课程, 有些学校还时断时续, 缺乏延续性。学术界对 该学科认知度依旧较低, 甚至有的学者不认可该门学科存在的必要性。目前已经形成的研究 才队多来自体育学, 学科背景、知识结构较为单一, 综合科研能力亟待加强。[5]虽经过多年 努力, 目前仍然没有形成严格意义上体育人类学自己的理论体系, 学科队伍的薄弱导致无法 快速充实与完善体育人类学的学科理论, 甚至有研究者认为, 近年来的中国体育人类学基础 理论研究基本处于停滞状态。从体育人类学学科长远发展需要出发, 急需打破学科壁垒, 加 强与人类学、民族学、社会学领域专家学者的交流合作, 主动寻求指导和支持, 进一步提高 体育人类学的整体理论研究水平，尽快建立中国体育人类学研究的基础理论体系。

\section{2 与相关学科间的关系模糊}

体育人类学研究者在现实中经常会被提出下面一系列问题: “体育人类学是什么? ”, “体育人类学与体育人文社会学是什么关系? ”, “体育人类学研究与民俗学研究的差别在 哪? ”等等。这些问题都暴露出在学科发展过程中没有厘清与相关学科的关系, 尤其是没有 正确处理好与民俗学、体育社会学、体育文化学、民族传统体育学等相关之间的关系, 没有 找准自身的学科定位。这在一定程度上导致体育人文社会学蓬勃发展, 而体育人类学只能偏 安于一隅, 在部分师范类和体育类高校中有为数不多的研究生招生方向。从人类学与社会学 的学科关系上而言, 人类学研究人类及其文化, 社会学研究人类社会。因此, 依据母学科的 知识关系, 体育人类学在研究范围上要比体育社会学更广泛, 至少两者之间应该是一种依次 递进、互为补充的关系, 但现实却并非如此。[6]从研究的视角看, 体育社会学关于体育活动 中的文化现象研究, 在某种程度上又与体育人类学研究的领域重复, 这就容易造成学科界限 的混淆不清。因此, 厘清体育人类学与相关学科间的关系, 也是学科发展的一大重要工作。 是通过已有体育人文社会学的学科基础来促进体育人类学的自身发展, 还是将相关研究以种 属关系统合到体育人类学的学科视域之中还有待进一步的探讨。

\section{3 “产学研”集合发展的问题}

当下各学科发展都十分重视“产学研”集合发展, 对于一个新兴学科的建设, 不能回避这 一现实问题。就目前我国体育人类学发展现状看来, 研究领域拓展不足, 研究的应用取向不 明显。纵览国际体育人类学研究, 研究视角和领域都特别宽泛且与时俱进。美国人类学研究 者不仅仅局限于传统的异文化、民族认同、宗教仪式等研究, 他们把目光放在了大型运动赛 事、体育明星、社会异化、互联网、大数据、游戏、虚拟平台等方面。 ${ }^{[7]}$ 而国内的体育人类 学目光依旧更多的聚焦于民族传统与区域 (村落) 体育的研究, 尽管这与我们国家的国情有 关, 但从研究的内容来看大多数依旧在探讨历史源头及文化嬗变。对比看来, 首先我们在研 
究视角和切入点上就比较单一, 深层次反映出来的问题则是学科发展的广泛性、纵深性还相 对不足。

在人类学的研究中, 如何实现研究成果转化, 也是所有研究者需要考虑的, 只有凸显了 研究的价值和意义, 才能推动学科的长足发展。事实上, 在中国面临现代化转型的巨变新时 代, 体育文化建设和发展领域出现的很多新现象新问题, 有待学术研究提供智力支持和理论 指导，而体育人类学在其中完全可以发挥积极而重要的作用。例如，体育活动参与社会治 理; 重塑民族传统体育文化形态; 民族传统体育文化的民族认同与世界认同; 全民健身与校 园体育文化建设; 跨区域、国界间的民族体育交流; 体育事业如何提升国家文化软实力; 休 闲体育产业的持续发展、旅游业与体育产业的融合发展等等, 这些应用取向的研究不仅能够 落实费孝通先生关于中国人类学“志在富民”的宏愿, 促进社会文化发展, 同时也是体育人类 学持续发展的不竭动力。我国历史文化悠久, 民族多元, 数千年积累了无数璀璨的文明, 当 前正值国家转型的关键时期, 体育人类学应该用更广泛的视角去拓展研究的对象, 特别是在 一些具有应用价值的领域上加大研究的力度, 形成自己的学科特色, 努力打造“以适应社会 发展、提高国民身体文化素质”为目标的应用体育人类学研究范式, 以“产学研”一体化为理 念, 不断加强学科建设, 同时也为学科发展注入新的生命力。

\section{致谢}

本文为云南省教育厅科学研究基金项目（2018JS280）、云南农业大学 2017 年教育教学 改革研究项目 (2017YAUJY122)阶段性成果之一。

\section{Reference:}

[1] Hu Xiaoming. Introduction to Sports Snthropology [J]. Sports and Science, 2000,21 (3): 4-9.

[2] William A. Haviland. Cultural Anthropology: The Human Challenge [M]. Beijing: Machinery Industry Press, 2014:356.

[3] Luke Lust. Invitation of Anthropology [M]. Beijing: Peking University Press, 2008: preface.

[4] Ni Yike, Hu Xiaoming. The Trend of Traditional National Sports [J]. Sports Science, 2014,34 (12):3-7.

[5] Xie Xiaoyan, Wu Yongcun, Xie Yuhui. Reflections on the Study of Sports Anthropology [J].Journal of Chengdu Sport Institute , 2014, 40 (12): 41-44.

[6] Zhou Daming. Reflections on the Orientation of Anthropology [J].Journal of Guangxi University for Nationalities (Philosophy and Social Sciences), 2012, 34 (1): 81-83.

[7] Tu Chuanfei. Research progress of American Sports Anthropology and Enlightenment to Chinese Sports Anthropology [J].Sports Science, 2017,37(1): 68-79. 